\title{
A Compact, Low-Profile Log-Periodic Meandered Dipole Array Antenna with an Artificial Magnetic Conductor
}

\author{
Oh Heon Kwon, Sungwoo Lee, Jong Min Lee, and Keum Cheol Hwang (iD \\ School of Electronic and Electrical Engineering, Sungkyunkwan University, Suwon 440-746, Republic of Korea \\ Correspondence should be addressed to Keum Cheol Hwang; khwang@skku.edu
}

Received 22 January 2018; Revised 5 April 2018; Accepted 10 May 2018; Published 28 June 2018

Academic Editor: María Elena de Cos Gómez

Copyright (c) 2018 Oh Heon Kwon et al. This is an open access article distributed under the Creative Commons Attribution License, which permits unrestricted use, distribution, and reproduction in any medium, provided the original work is properly cited.

\begin{abstract}
A compact and low-profile log-periodic meandered dipole array (LPMDA) antenna with an artificial magnetic conductor (AMC) is proposed. For compactness, a meander line configuration is implemented with dipole elements and optimized using a genetic algorithm (GA) to realize the LPMDA antenna. As a result, a size reduction of approximately $30 \%$ is achieved as compared to a conventional log-periodic dipole array antenna. To enhance the gain characteristics, the AMC ground plane configuration is realized with $9 \times 9$ unit cells for the LPMDA antenna. Two prototypes of the proposed LPMDA antennas with and without an AMC are fabricated and measured to verify its performance. The measured $-10 \mathrm{~dB}$ reflection ratio bandwidths are $2.56: 1$ $(0.85-2.18 \mathrm{GHz})$ and $2.34: 1(0.92-2.16 \mathrm{GHz})$ for the proposed LPMDA antennas with and without the AMC, respectively. The gain at the main beam direction within the operating frequency bandwidth is significantly improved from $3.94-7.17 \mathrm{dBi}$ to 7.86-10.01 dBi by applying the AMC.
\end{abstract}

\section{Introduction}

Since Isbell proposed the log-periodic dipole array (LPDA) antenna in the 1960s [1], this antenna has been utilized in various communication systems due to its broadband impedance bandwidth, high gain, and simple design. The length of the longest dipole element of a typical LPDA antenna should be equal to half of the wavelength at the lowest operating frequency. Due to this property, it is not suitable for applications which have area limitations, such as aircraft and vehicle platforms. To apply the LPDA antenna to these platforms, the antenna should be miniaturized. For miniaturization of the LPDA antenna, several techniques have been introduced [2-5]. A fractal tree LPDA antenna was suggested by Wang et al. [2], and a Koch-shaped fractal was applied to an LPDA antenna by Anagnostou et al. [3]. In other studies, a T-shaped top-loaded LPDA antenna [4] and a compact dielectric-loaded LPDA antenna [5] were introduced by Chen et al. and Chang et al., respectively. These antennas are more compact than typical LPDA antennas, but they have lower gain characteristics. To improve the gain characteristics, additional components were applied to a miniaturized LPDA antenna $[6,7]$. Hsu and Huang applied parasitic elements as a director to a Koch-shaped LPDA antenna [6]. However, their gain characteristics remained lower than those of a typical LPDA antenna. In another study by Haraz et al. [7], an LPDA antenna with dielectric lenses was introduced. The gain of this antenna is higher than that of a typical antenna, but it is bulky and difficult to fabricate.

Metamaterials, which have properties that do not exist in nature, are widely applied in antenna engineering $[8,9]$. Specifically, one type of metamaterial, known as an artificial magnetic conductor (AMC, also referred to as a reactiveimpedance surface or a high-impedance surface), which has a periodic structure, was proposed by Sievenpiper et al. [10]. An AMC is frequently used to miniaturize antennas and to achieve a gain enhancement [11-13]. Similar to a perfect magnetic conductor (PMC), the image current is formed in-phase on the surface of the AMC, and only the 


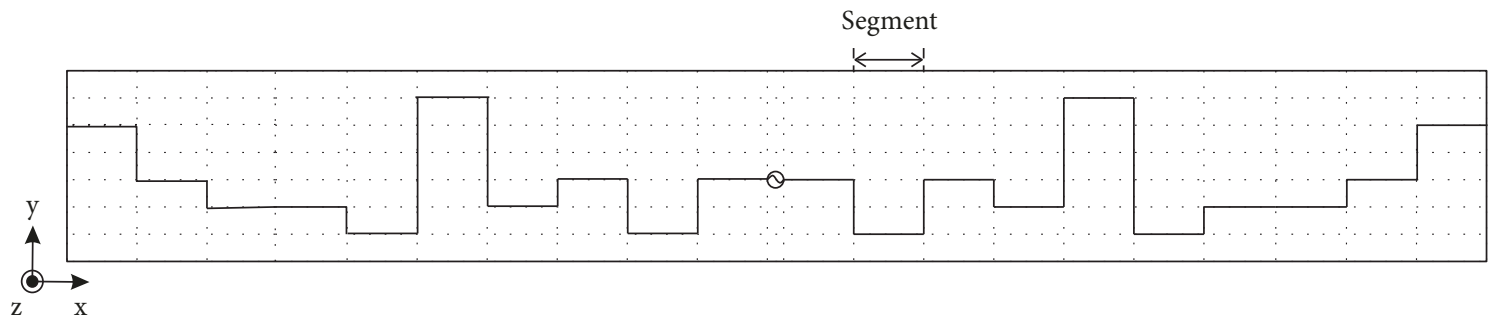

FIGURE 1: Design concept for a meandered dipole element with a fixed grid structure.

surface impedance of the AMC is reactive. With these two properties of the AMC, the antenna can be miniaturized [14]. The gain of the antenna can be improved by the AMC, which has in-phase reflection phase characteristics within a specific frequency band. Owing to this characteristic, the AMC can operate as a reflector when located closer to the antenna than a general PEC reflector would be [15].

Previously, the log-periodic microstrip patch antenna, which consists of square patch elements, was investigated [16]. The log-periodic antenna was miniaturized and the operating bandwidth was enhanced by applying the AMC. Despite the fact that the AMC was applied, the gain characteristics of the proposed antenna were decreased as compared to those of an antenna without an AMC.

In this paper, a compact and low-profile log-periodic meandered dipole array (LPMDA) antenna with an AMC is proposed. First, the longest element of the LPMDA was designed based on the meander line configuration and was optimized using a binary genetic algorithm (GA). Second, based on the designed longest meandered element, the LPMDA was formed and its performance was compared to that of a typical LPDA antenna. Finally, the AMC, which has a reflection phase bandwidth covering the operating bandwidth of the LPMDA antenna, was applied as the reflector of the LPMDA to enhance the gain. ANSYS highfrequency structure simulator (HFSS) software was used to conduct all of the simulations in this study. The proposed antennas, with and without an AMC, were experimentally verified. The design of the antenna with the AMC is introduced in Section 2. Section 3 presents a comparison between the measured and simulated results. Finally, the conclusion is given in Section 4.

\section{Antenna Design}

The design process of an LPDA can start with the use of the formula by Carrel [17]:

$$
\begin{aligned}
& \tau=\frac{l_{n}}{l_{n-1}}=\frac{w_{n}}{w_{n-1}}=\frac{d_{n}}{d_{n-1}}, \\
& \sigma=\frac{d_{n}}{2 l_{n-1}},
\end{aligned}
$$

where $l_{n}$ and $w_{n}$ are the length and width of the $n$th dipole element, respectively; $d_{n-1}$ is the distance between $n$th and $(n-1)$ th dipole elements; and $n$ is the number of dipole elements of LPDA antenna. The extension factor $(\tau)$, spacing factor $(\sigma)$, and the number of dipole elements $(n)$ are set to $0.85,0.15$, and 7 , respectively.

Figure 1 depicts the design concept for the meandered dipole element of the longest element of the LPMDA. This design concept was recently developed by Bayraktar et al. [18]. A fixed grid of a reduced length is introduced, and each dipole arm is divided into ten segments. Each segment lies on one column of the grid, and two adjacent segments are connected through a vertical line. The binary GA is then used to determine the optimal position of each segment in the columns to form a meander line configuration that can exhibit performance comparable to that of its conventional full-size counterpart. During the implementation of the binary GA, the position of each segment is encoded into three bits. Therefore, there are 30 bits used to realize the meandered dipole element. Subsequently, using (1), a seven-element LPMDA is formed based on the longest meandered dipole element, as shown in Figure 2. The spacing between two adjacent dipole elements is also optimized by the binary GA, during which each spacing distance is encoded into eight bits. The total number of bits to be used for the optimization is 78 bits. The binary GA is developed with MATLAB programming linked to HFSS through an HFSS scripting interface. One hundred iterations, a population of 20 , and a mutation rate of 0.1 with the single-point crossover scheme is used. During the simulation with HFSS, each segment of the longest dipole element is modeled by a rectangular patch $3.5 \times 7.8 \mathrm{~mm}^{2}$ in size. The LPMDA is designed on a Taconic RF-35 substrate with a dielectric constant of 3.5, a thickness of $1.52 \mathrm{~mm}$, and a loss tangent of 0.0018 . The dipole elements of the LPMDA antenna located on the upper and lower side of the substrate are indicated here in gray and white, respectively. The designed antenna is fed at one end of the parallel transmission strip line near the shortest dipole element. The other end of the parallel transmission strip line is in an open state. Here, $L, W$, and $w_{f}$ indicate the length and width of the substrate of the LPMDA antenna and the parallel transmission stripline width, respectively. Table 1 summarizes the optimized design parameters of the LPMDA.

For a comparison of the LPMDA antenna, a typical LPDA antenna with an identical operating bandwidth of $2: 1(1-2 \mathrm{GHz})$ and with an $8 \mathrm{dBi}$ gain was designed using 


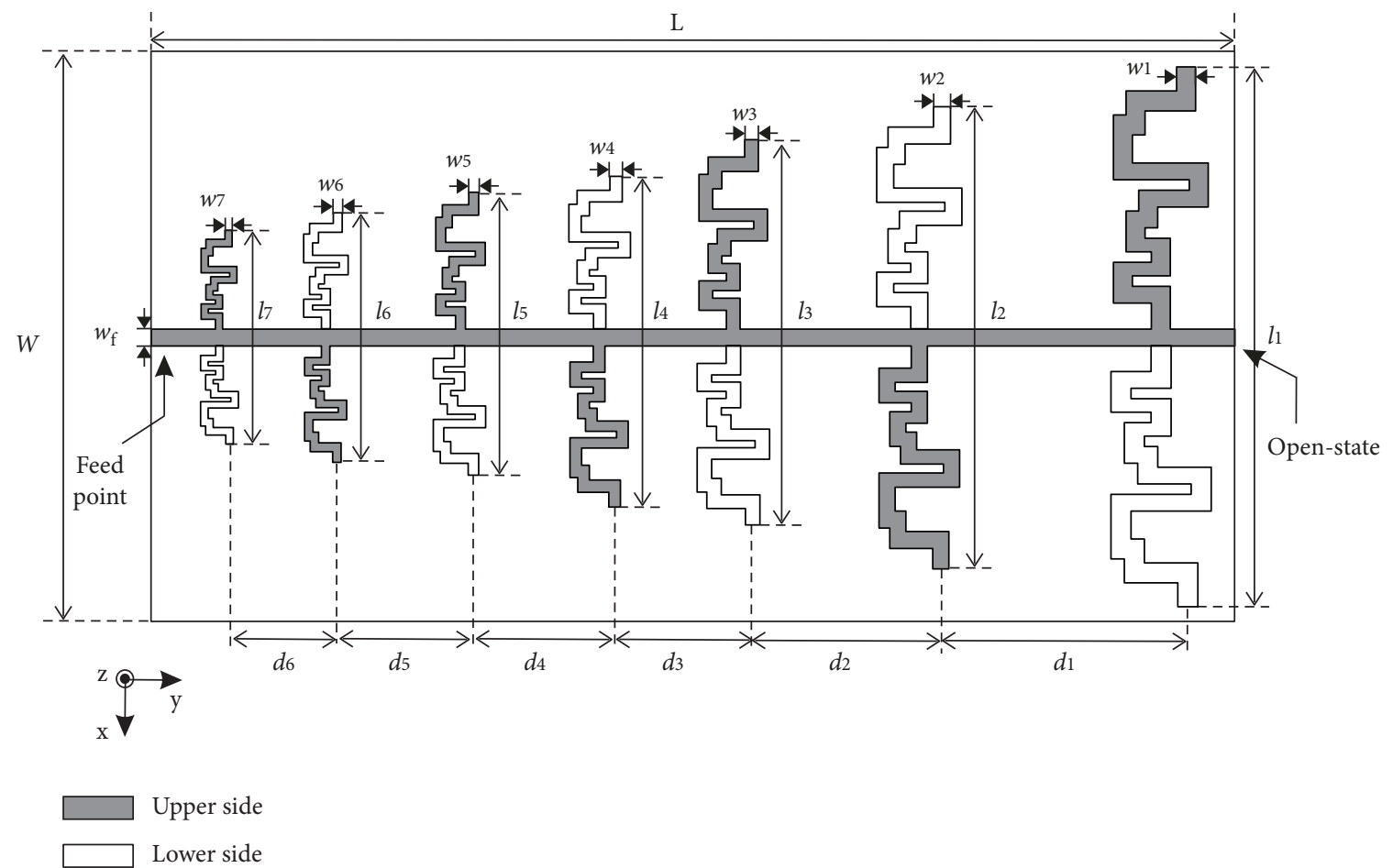

Figure 2: Geometry of the log-periodic meandered dipole array (LPMDA) antenna.

TABle 1: Optimal parameters of log-periodic meandered dipole array (LPMDA) antenna.

\begin{tabular}{lccccc}
\hline Parameters & $\begin{array}{c}\text { Values } \\
(\mathrm{mm})\end{array}$ & Parameters & $\begin{array}{c}\text { Values } \\
(\mathrm{mm})\end{array}$ & Parameters & $\begin{array}{c}\text { Values } \\
(\mathrm{mm})\end{array}$ \\
\hline$W$ & 120 & $L$ & 203 & $d_{1}$ & 45.96 \\
$w_{1}$ & 3.5 & $l_{1}$ & 96.0 & $d_{2}$ & 35.46 \\
$w_{2}$ & 3.01 & $l_{2}$ & 82.11 & $d_{3}$ & 25.64 \\
$w_{3}$ & 2.56 & $l_{3}$ & 70.24 & $d_{4}$ & 22.39 \\
$w_{4}$ & 2.18 & $l_{4}$ & 60.16 & $d_{5}$ & 25.63 \\
$w_{5}$ & 1.85 & $l_{5}$ & 51.58 & $d_{6}$ & 20.29 \\
$w_{6}$ & 1.57 & $l_{6}$ & 44.29 & & \\
$w_{7}$ & 1.33 & $l_{7}$ & 38.10 & & \\
$w_{f}$ & 3.0 & & & & \\
\hline
\end{tabular}

the formula devised by Carrel on Taconic RF-35. The length of the longest dipole element of the typical LPDA antenna is set to $118 \mathrm{~mm}$. Moreover, the extension factor $(\tau)$, spacing factor $(\sigma)$, and the number of dipole elements $(n)$ are identical to those of the LPMDA antenna. The reflection coefficients of the LPDA antenna and the LPMDA antenna are shown in Figure 3. The simulated $-10 \mathrm{~dB}$ reflection ratio bandwidths are 1.95:1 (0.99-1.94 GHz) and 2.31:1 (0.93$2.15 \mathrm{GHz}$ ), respectively. It could be confirmed that the antenna is miniaturized by $30 \%$ from $0.49 \times 0.66 \lambda_{L}^{2}$ to $0.37 \times 0.62 \lambda_{L}^{2}$ by applying a meander line to the dipole

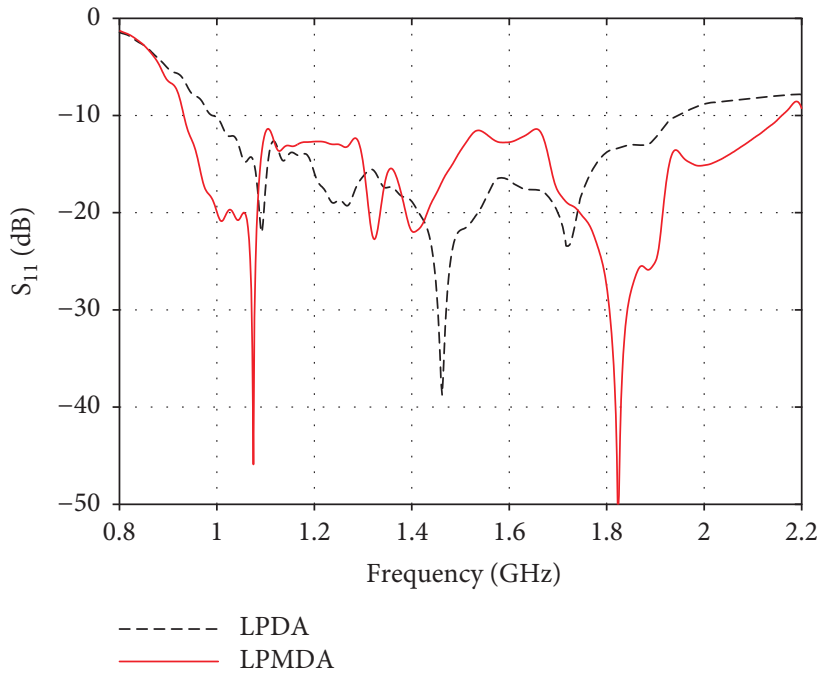

FIgURE 3: Simulated reflection coefficients of a typical log-periodic dipole array (LPDA) antenna and of the LPMDA antenna.

element $\left(\lambda_{L}\right.$ indicates the wavelength of the lowest operating frequency of the antenna). In addition, the LPMDA antenna has a wider operating ratio bandwidth than the LPDA antenna. Comparisons of the far-fields and the gains of the LPMDA and LPDA antennas are conducted. The radiation patterns of the LPDA and LPMDA antennas on the $y z$-plane at $1 \mathrm{GHz}, 1.3 \mathrm{GHz}$, and $1.8 \mathrm{GHz}$ are illustrated in Figure 4. It is evident that both antennas exhibit a 


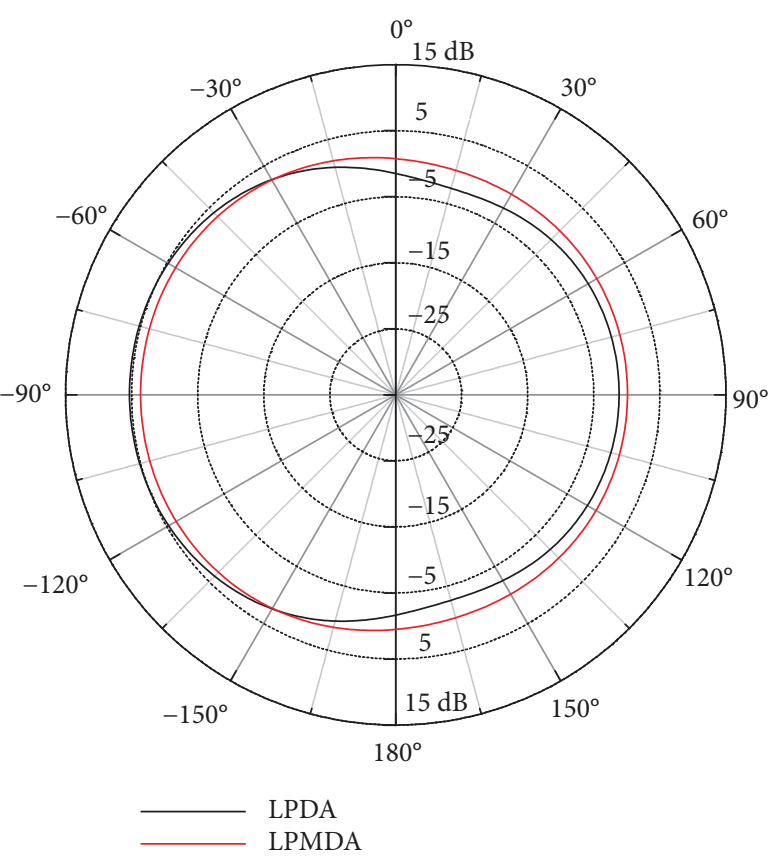

(a)

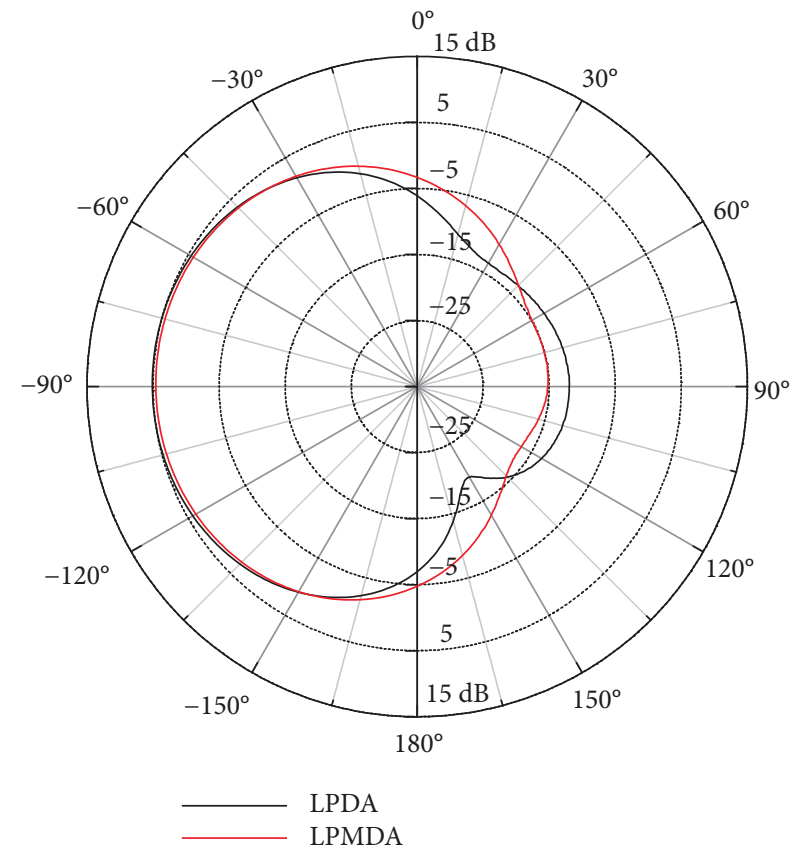

(b)

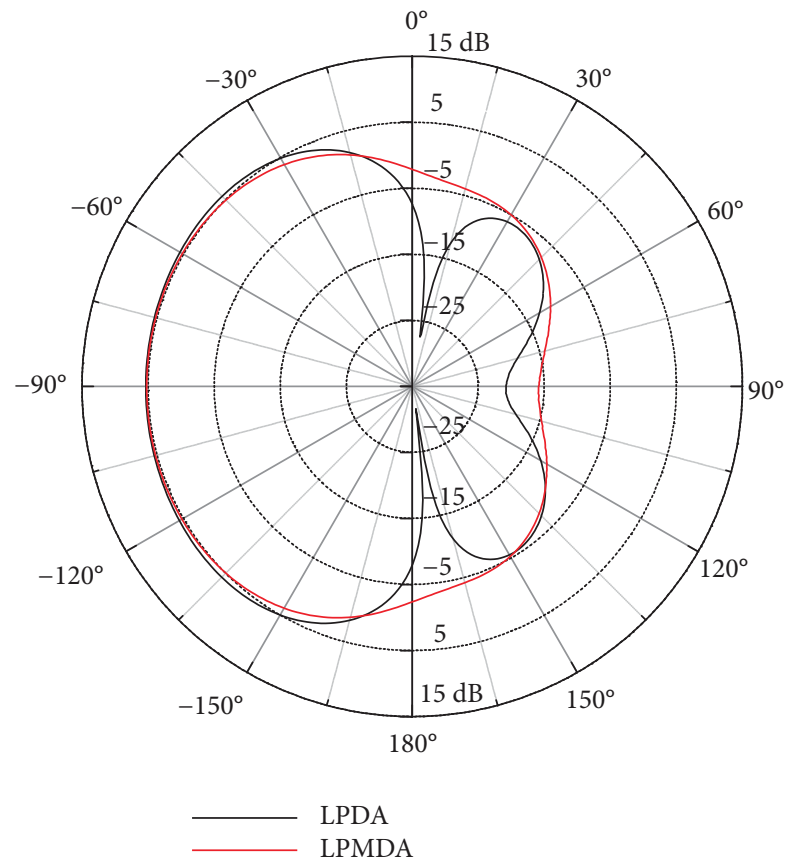

(c)

FIGURE 4: Simulated radiation patterns of the LPDA antenna and LPMDA antenna on the $y z$-plane at (a) $1 \mathrm{GHz}$, (b) $1.3 \mathrm{GHz}$, and (c) $1.8 \mathrm{GHz}$.

directional radiation pattern at all three frequencies. It is also noted the main beam points toward the feed location. Figure 5 shows the simulated gains of the LPDA and LPMDA antennas. As observed, when applying the meander line to achieve a compact size, the gain of the LPMDA antenna is lower than that of the LPDA antenna.

Figures 6(a) and 6(b) illustrate the geometry of the unit cell of the proposed AMC and the boundary condition of the AMC when it was simulated, respectively. The unit structure is composed of two $1.52 \mathrm{~mm}$ thick RF-35 substrates with a side length of $W=30 \mathrm{~mm}$ and a distance $h_{a}=40 \mathrm{~mm}$. On the upper surface of the above-placed substrate, a square conductor with a side length of $\alpha=18 \mathrm{~mm}$ is located. The ground plane is located on the lower side of the lower substrate. In order to implement the periodic boundary condition, the walls perpendicular to the $x$ - 


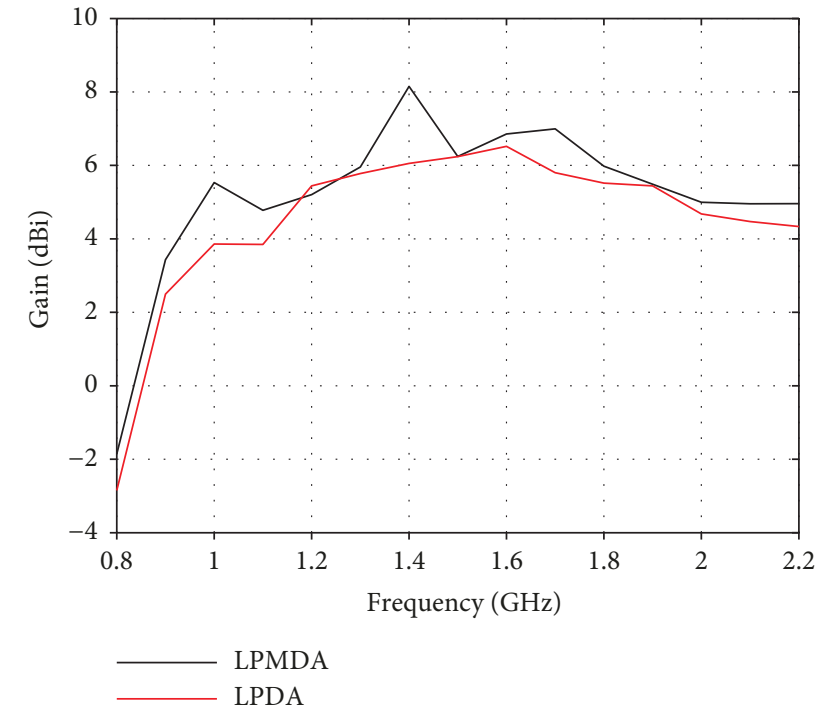

FIGURE 5: Simulated gains of the LPDA antenna and LPMDA antenna in the main beam direction.

and $y$-axes are defined as PMC and PEC, respectively. To compute the reflection phase on the AMC surface, the waveport, which is located along the $z$-axis, propagates the uniform wave.

Figure 7 shows the reflection phase of the proposed unit cell of the AMC. The $\pm 90^{\circ}$ reflection phase ratio bandwidth, which exhibits in-phase reflection phase characteristics, is 2.88:1 $(0.71-2.05 \mathrm{GHz})$. Referring to Figure 3, it can be confirmed that the reflection phase bandwidth of the unit cell of the AMC covers the operating frequency bandwidth of the LPMDA antenna.

In the design process of the AMC, several major parameters are investigated. These are the distance between two dielectric substrates $h_{a}$, the width of AMC patch $a$, and the width of AMC cell $W$. The simulated results of the refection phases are illustrated in Figure 8. It is found that the reflection phase bandwidth is shifted down to a lower frequency range when each of the parameters $\left(h_{a}, a\right.$, or $\left.W\right)$ is increased. In order to apply the AMC to the LPMDA antenna, the reflection phase bandwidth of the AMC should fit the operating frequency band of the antenna. Therefore, the parameters $h_{a}, a$, and $W$ are finally set to $40 \mathrm{~mm}, 18 \mathrm{~mm}$, and $30 \mathrm{~mm}$, respectively, so that the optimized AMC exhibits a reflection phase bandwidth of $0.71-2.05 \mathrm{GHz}$.

In order to demonstrate the effectiveness of the proposed AMC structure, a comparison is conducted of the reflection phases of the proposed AMC structure, a conventional AMC structure with one substrate (configuration 1) and a conventional AMC structure with two stacked substrates (configuration 2). The configurations of these AMC structures and the simulated reflection phases are shown in Figure 9. Note that the parameters $W, a$, and $h$ of these AMC structures are identical for a fair comparison and they are set to $40 \mathrm{~mm}, 18 \mathrm{~mm}$, and $1.52 \mathrm{~mm}$, respectively. It is observed that the conventional AMC structure (configuration 1) exhibits a

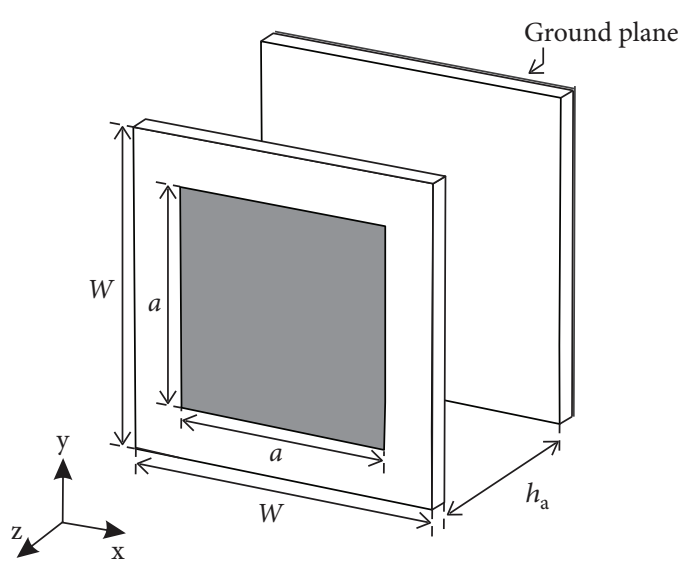

(a)

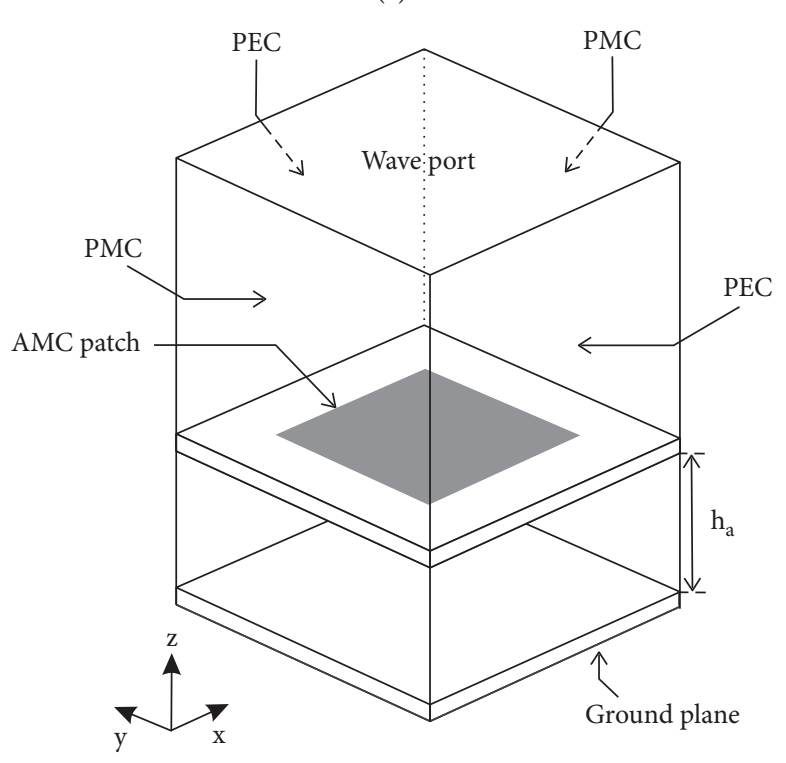

(b)

FIgURE 6: Geometry of the unit cell of the proposed artificial magnetic conductor (AMC): (a) perspective view and (b) boundary condition of the AMC in the simulation.

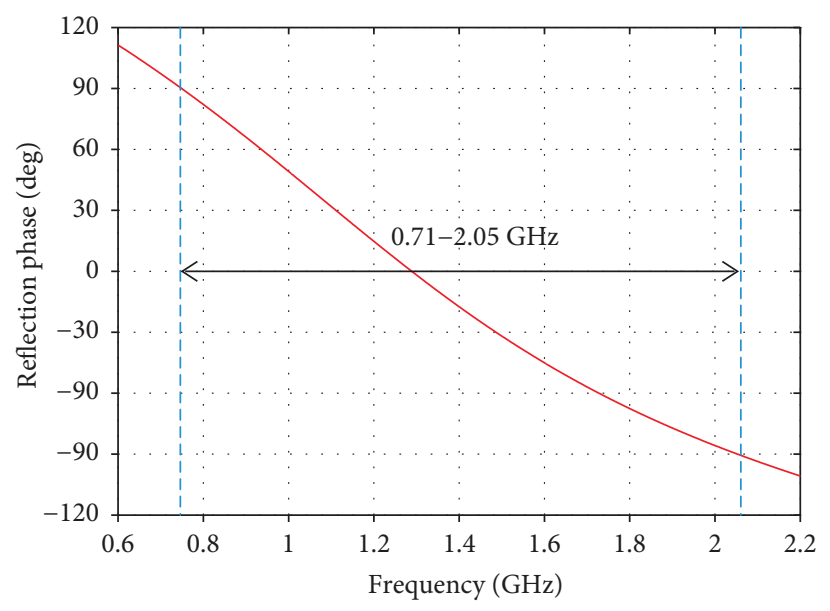

Figure 7: Simulated reflection phase of the unit cell of the proposed AMC. 


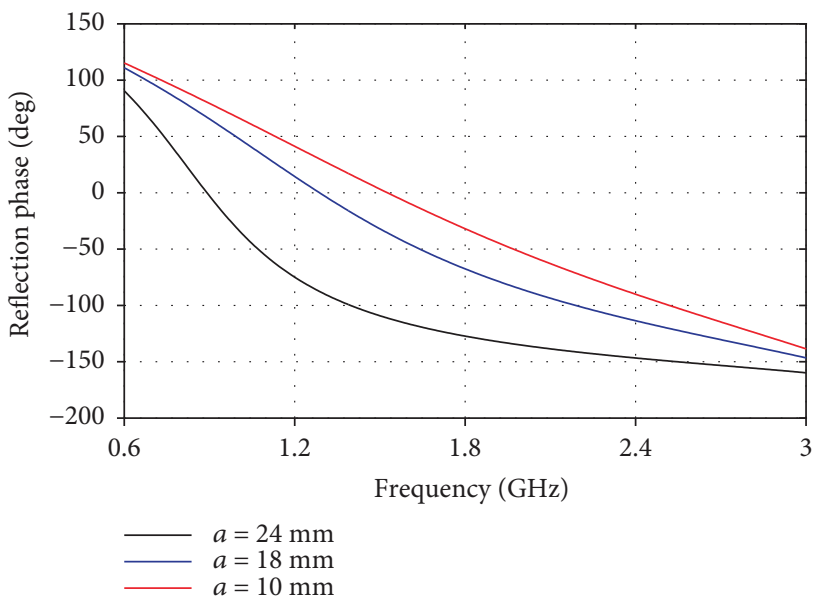

(a)

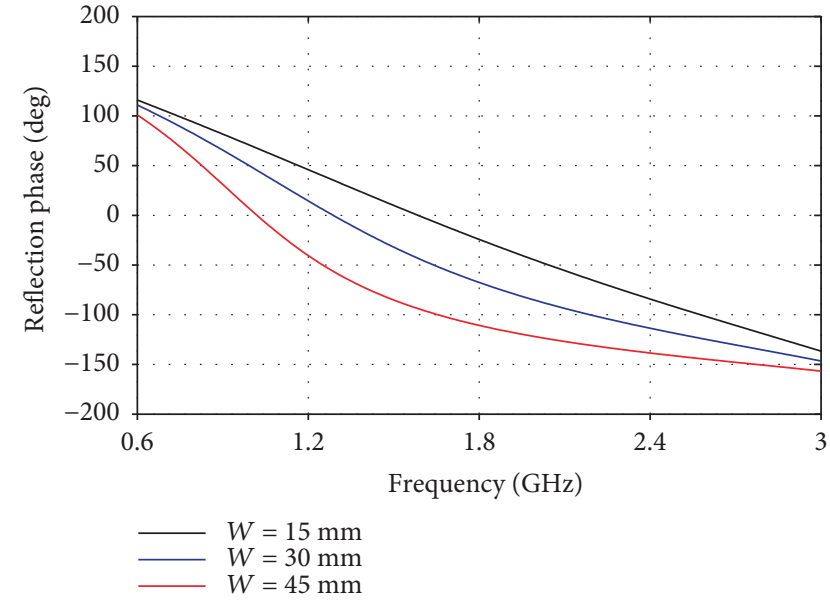

(b)

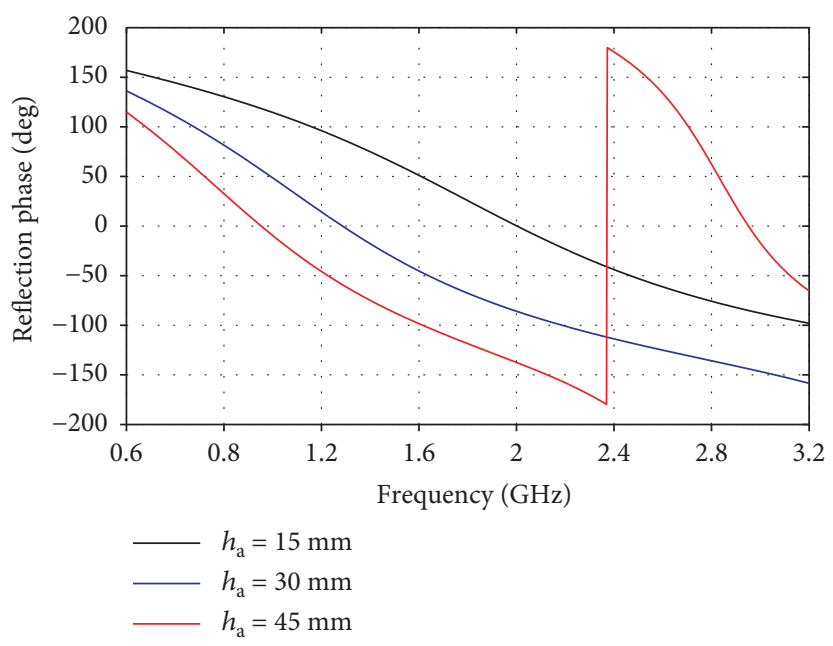

(c)

Figure 8: Simulated reflection phases of AMC with changes in (a) $a$, (b) $W$, and (c) $h_{a}$.

narrow $\pm 90^{\circ}$ reflection phase ratio bandwidth of $1.05: 1$ (4.05-4.27 GHz). By using two stacked substrates, the reflection phase bandwidth is slightly shifted down to 3.64$4.13 \mathrm{GHz}(1.13: 1)$. These bands are very high as compared to the operating frequency bandwidth of the antenna, which is $0.93-2.15 \mathrm{GHz}$. To operate in lower frequency band, we can increase the size $h$ of the AMC unit cell. However, as the reflection phase bandwidth of the conventional AMC is quite narrow, it is difficult to match the operating frequency band of the LPMDA antenna $(0.93-2.15 \mathrm{GHz}, 2.31: 1)$. In contrast, the proposed AMC structure, which is designed on two different substrates separated by an air gap, has a wide reflection phase ratio bandwidth of $2.73: 1(0.75-2.05 \mathrm{GHz})$. This frequency band nearly matches the operating frequency band of the LPMDA antenna.

The LPMDA antenna with the AMC is shown in Figure 10. This antenna is fed by a coaxial cable, as shown in Figure 10. The AMC applied to the LPMDA antenna is composed of $9 \times 9$ unit cells. The designed AMC is located below the antenna at a distance Gap equal to $5 \mathrm{~mm}$.

\section{Experimental Results and Discussion}

Based on the design parameters presented in the previous section, a prototype of the proposed antenna with the AMC was fabricated, as shown in Figure 11. Nylon posts with a dielectric constant of 3.2 are used to support the LPMDA antenna above the AMC and to form an air gap between the two dielectric substrates that constitute the AMC structure. The proposed antenna was excited by a coaxial cable, as shown in Figure 11.

An Agilent 8510C network analyzer was used to measure the reflection coefficients of the fabricated antennas. Figure 12 presents the simulated and measured reflection coefficients of the LPMDA antennas with and without the AMC. The simulated and measured results are in good agreement. The simulated and measured reflection ratio bandwidths of the LPMDA antenna without the AMC are $2.31: 1(0.93-2.15 \mathrm{GHz})$ and $2.34: 1(0.92-2.16 \mathrm{GHz})$, respectively. On the other hand, the corresponding simulated and measured reflection ratio bandwidths of the LPMDA antenna with the AMC are 2.54:1 (0.85-2.16 GHz) and 


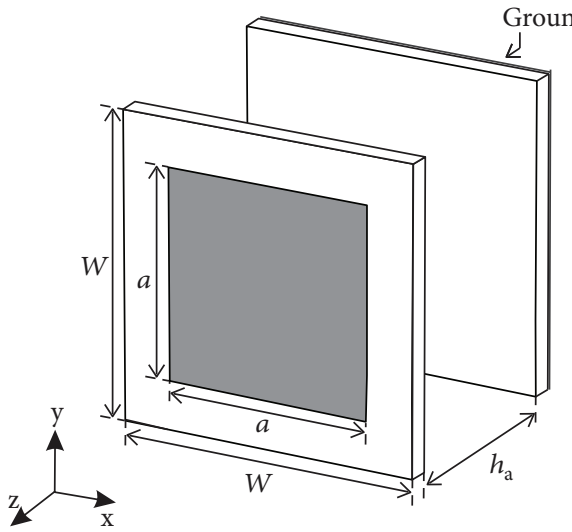

The proposed AMC

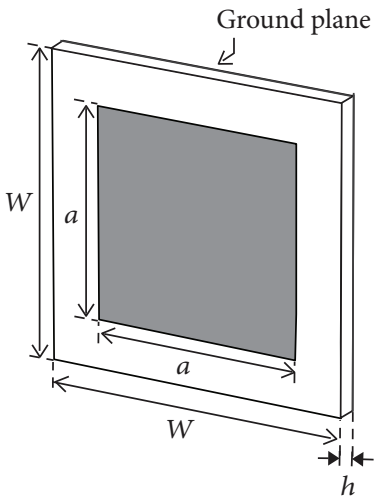

Configuration 1

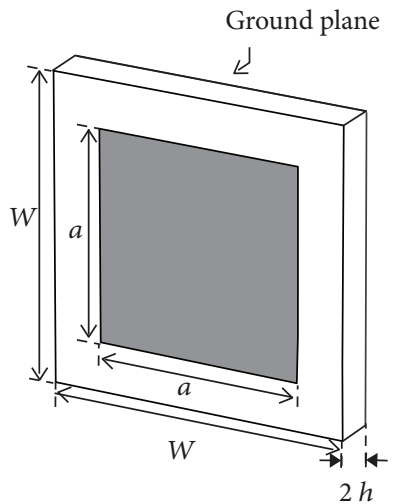

Configuration 2

(a)

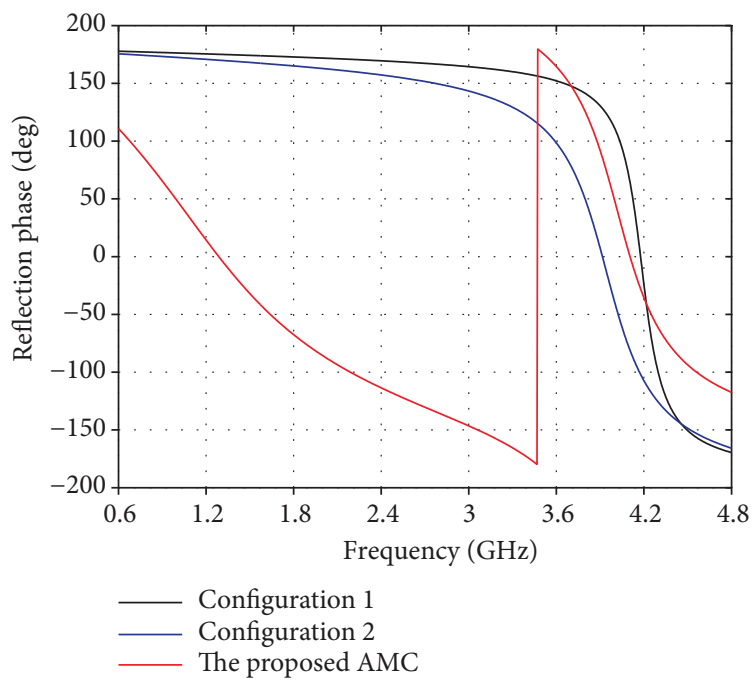

(b)

Figure 9: (a) Geometry of the unit cells of the AMC structures and (b) simulated reflection phases.

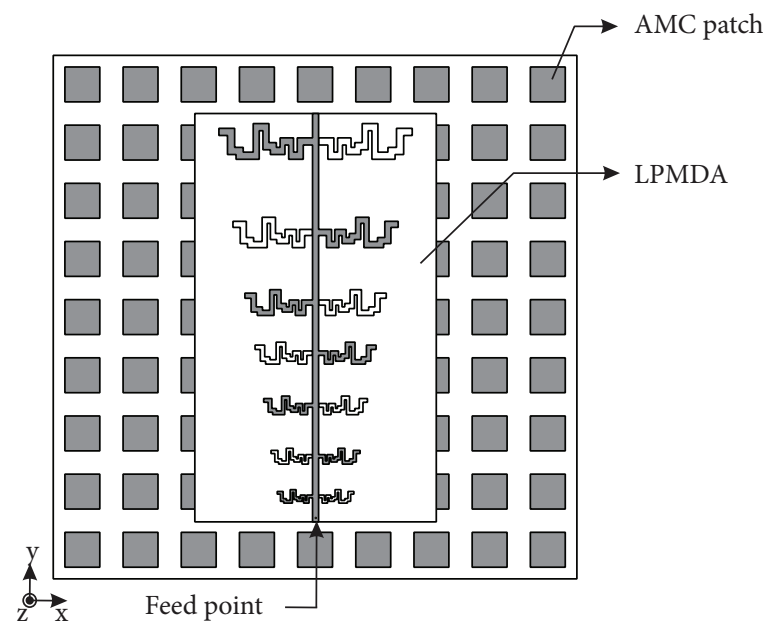

(a)

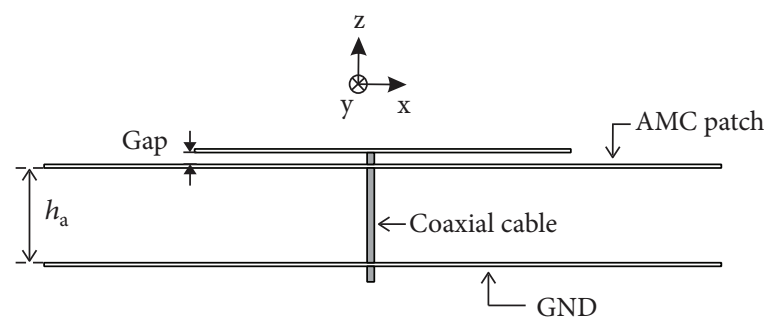

(b)

FIGURE 10: Geometry of the proposed antenna with the AMC: (a) top view and (b) side view. 


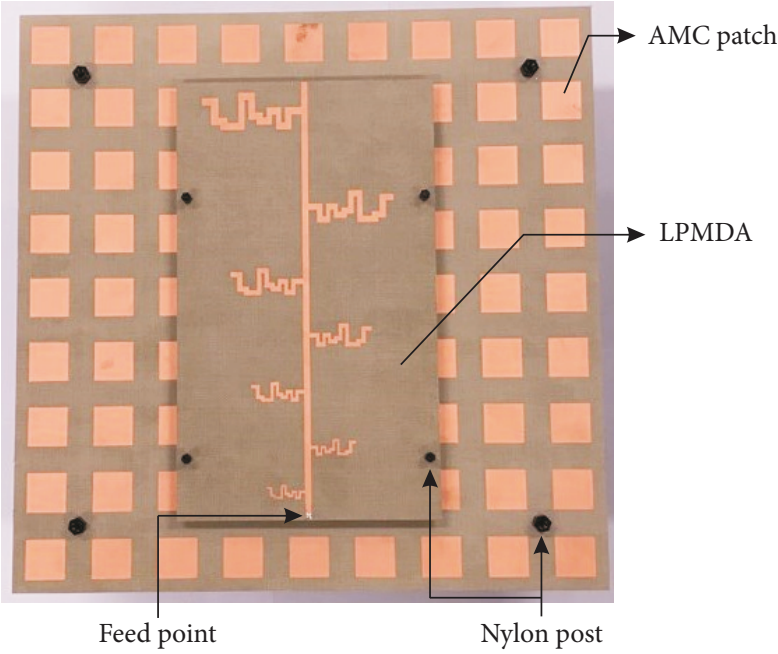

(a)

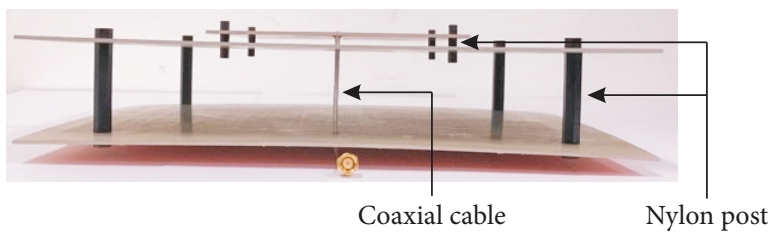

(b)

FIgURE 11: Photograph of the fabricated antenna with the AMC: (a) top view and (b) side view.

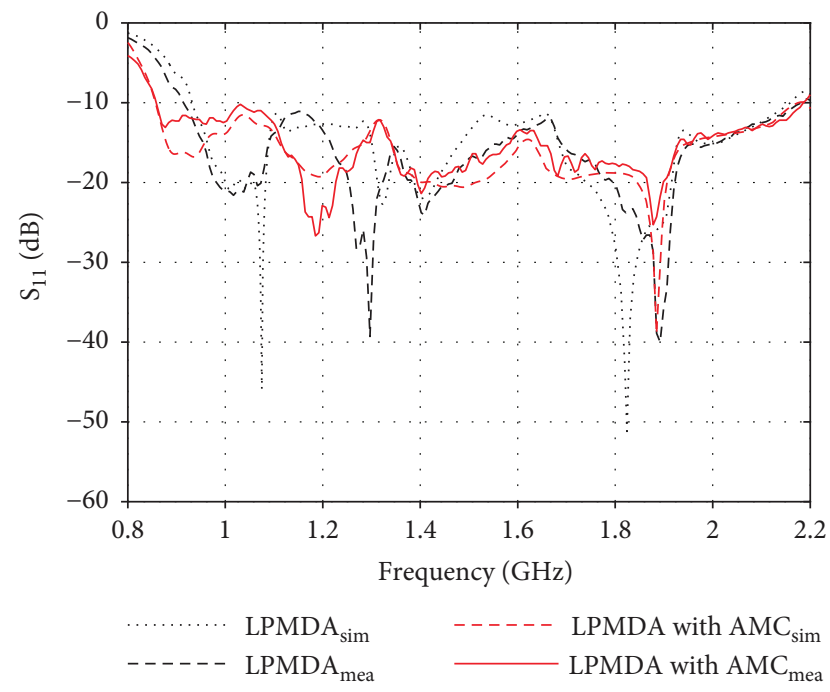

Figure 12: Simulated and measured reflection coefficients of the proposed antenna.

2.56: $1(0.85-2.18 \mathrm{GHz})$, respectively. The lowest operating frequency of the antenna is lowered from 0.92 to $0.85 \mathrm{GHz}$ by applying the AMC. Furthermore, the operating frequency bandwidth of the antenna is increased.

The simulated and measured radiation patterns on the $y z$-plane are shown in Figure 13. The main beam direction of the LPMDA antenna without the AMC is formed at $\theta=-90^{\circ}$. However, the main beam direction of the LPMDA antenna with the AMC is tilted. Owing to its in-phase reflection characteristics, the AMC serves as the reflector even if it is positioned closer to the antenna than a general conductor reflector, which usually requires a distance of at least $0.25 \lambda_{L}$. In the proposed LPMDA antenna with the AMC structure, this distance is only in the region of $0.14 \lambda_{L}$. This is also confirmed by the enhanced gain characteristics of the main beam direction of the antenna, as shown in Figure 14. The gain of the LPMDA antenna without the AMC varies from 3.94 to $7.17 \mathrm{dBi}$ within the operating frequency bandwidth. In contrast, in the main beam direction, the gain of the LPMDA antenna with the AMC varies from 7.86 to $10.01 \mathrm{dBi}$ within the operating frequency band. As the AMC structure is applied, a gain enhancement is achieved but the total size of the proposed antenna is also increased. However, from an application standpoint, the proposed antenna is designed for applications such as mobile vehicles and aircrafts, in which the antenna is implemented into a platform with a very large conductor. Due to its in-phase reflection characteristics, the AMC structure, which is considered as a part of the platform, can reduce the distance between the antenna and the platform conductor, therefore realizing a low-profile antenna without deteriorating the performance capabilities.

\section{Conclusion}

A compact and low-profile LPMDA antenna with an AMC was proposed and verified in this paper. The meander line was applied to the dipole elements of the LPMDA antenna. The AMC was designed to have a reflection phase bandwidth that covers the operating bandwidth of the LPMDA antenna. As a result, the measured $-10 \mathrm{~dB}$ reflection ratio bandwidth of the proposed antenna with the AMC is 2.56:1 (0.85$2.18 \mathrm{GHz}$ ). The gain in the main beam direction ranges from 7.86 to $10.01 \mathrm{dBi}$ within the operating frequency bandwidth. Compared to a typical LPDA antenna, the antenna is $30 \%$ miniaturized from $0.49 \times 0.66 \lambda_{L}^{2}$ to $0.42 \times 0.57 \lambda_{L}^{2}$, and high gain characteristics and a low profile are realized due to the use of the AMC. Because the proposed antenna with the AMC is successfully miniaturized, has a high gain, 


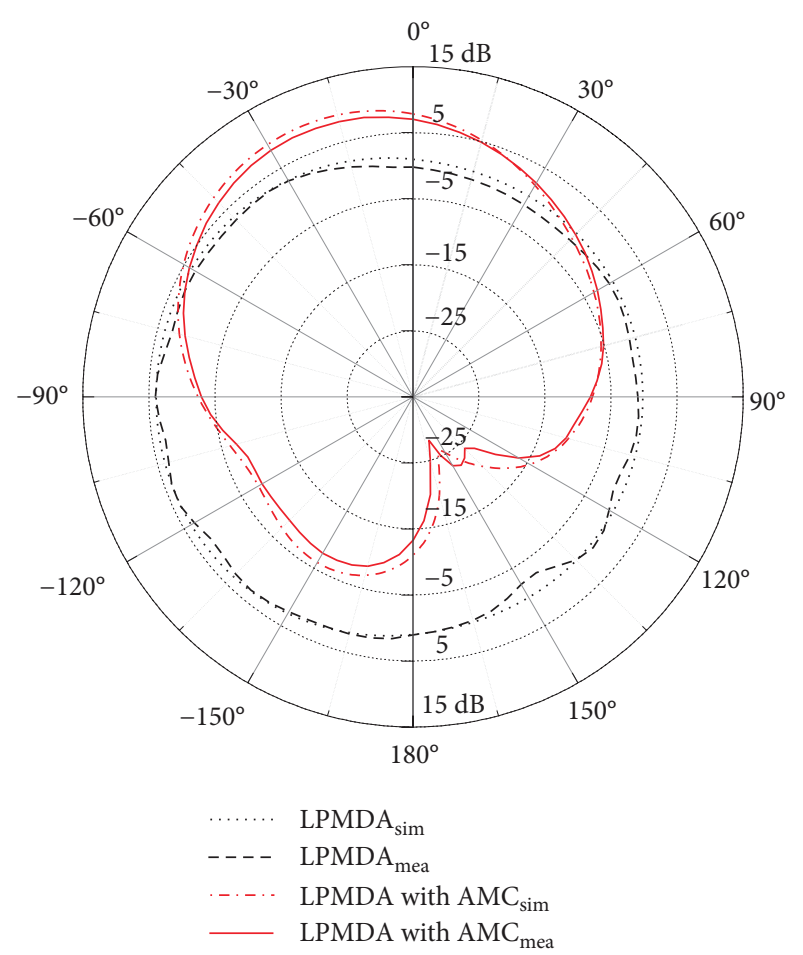

(a)

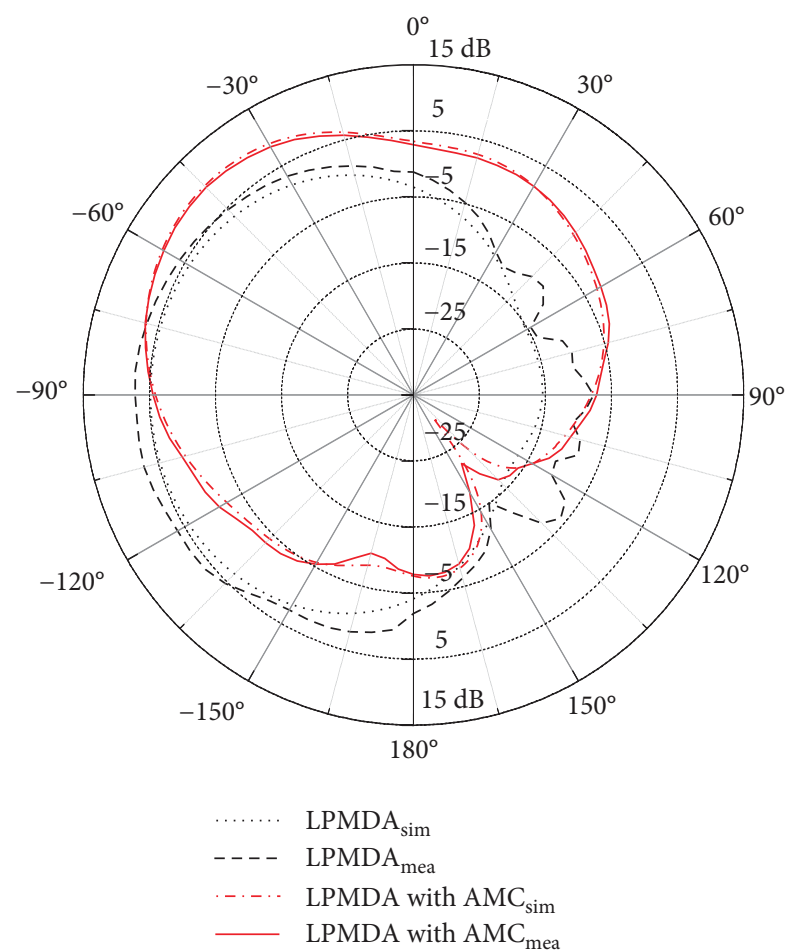

(b)

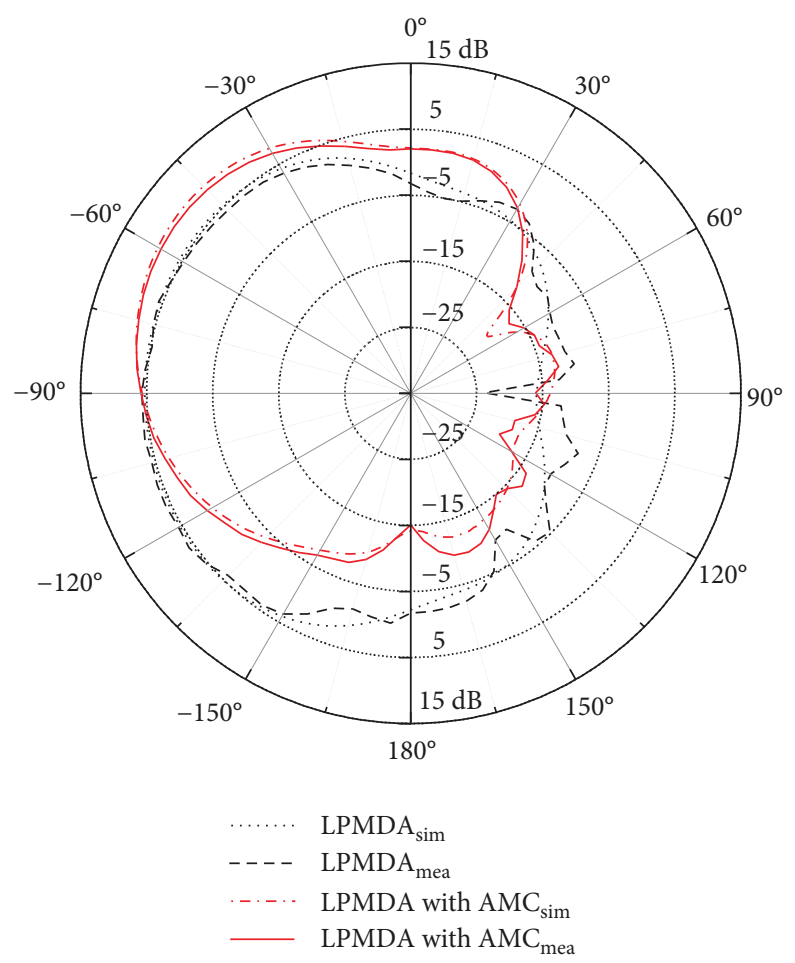

(c)

Figure 13: Simulated and measured radiation patterns on the $y z$-plane at (a) $1 \mathrm{GHz}$, (b) $1.3 \mathrm{GHz}$, and (c) $1.8 \mathrm{GHz}$.

and has a low profile, this antenna is suitable for applications such as aircraft and cars, where the antenna used can affect the aerodynamic performance outcomes.
In addition, although the main beam direction of the LPMDA antenna with the AMC is tilted, it will not influence communications. 


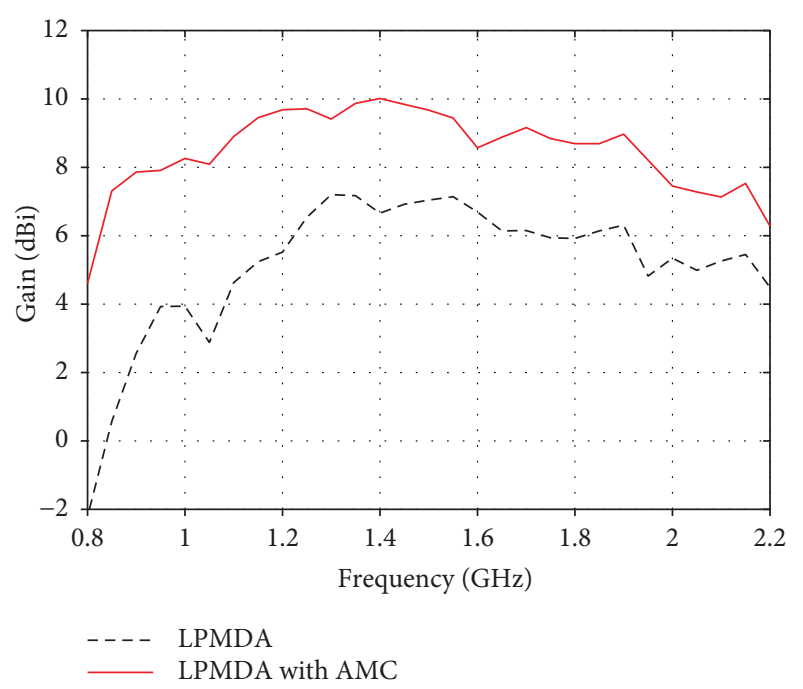

FIGURE 14: Measured gains of the proposed antennas in the main beam direction.

\section{Data Availability}

The data used to support the findings of this study are available from the corresponding author upon request.

\section{Conflicts of Interest}

The authors declare that they have no conflicts of interest.

\section{Acknowledgments}

This work has been supported by the Future Combat System Network Technology Research Center Program of the Defense Acquisition Program Administration and Agency for Defense Development (UD160070BD).

\section{References}

[1] D. Isbell, "Log periodic dipole arrays," IRE Transactions on Antennas and Propagation, vol. 8, no. 3, pp. 260-267, 1960.

[2] B. Wang, A. Chen, and D. Su, "An improved fractal tree logperiodic dipole antenna," in 2008 Asia-Pacific Symposium on Electromagnetic Compatibility and 19th International Zurich Symposium on Electromagnetic Compatibility, pp. 831-834, Singapore, Singapore, May 2008.

[3] D. E. Anagnostou, J. Papapolymerou, M. M. Tentzeris, and C. G. Christodoulou, "A printed log-periodic Koch-dipole array (LPKDA)," IEEE Antennas and Wireless Propagation Letters, vol. 7, pp. 456-460, 2008.

[4] J. Chen, J. Ludwig, and S. Lim, "Design of a compact log-periodic dipole array using T-shaped top loadings," IEEE Antennas and Wireless Propagation Letters, vol. 16, pp. 1585-1588, 2017.

[5] L. Chang, S. He, J. Q. Zhang, and D. Li, "A compact dielectricloaded log-periodic dipole array (LPDA) antenna," IEEE Antennas and Wireless Propagation Letters, vol. 16, pp. 27592762, 2017.
[6] H.-T. Hsu and T.-J. Huang, "A Koch-shaped log-periodic dipole array (LPDA) antenna for universal ultra-highfrequency (UHF) radio frequency identification (RFID) handheld reader," IEEE Transactions on Antennas and Propagation, vol. 61, no. 9, pp. 4852-4856, 2013.

[7] O. M. Haraz, S. A. Alshebeili, and A.-R. Sebak, "Low-cost high gain printed log-periodic dipole array antenna with dielectric lenses for V-band applications," IET Microwaves, Antennas \& Propagation, vol. 9, no. 6, pp. 541-552, 2015.

[8] D. Marathe and K. Kulat, "A compact triple-band negative permittivity metamaterial for $\mathrm{C}, \mathrm{X}$-band applications," International Journal of Antennas and Propagation, vol. 2017, Article ID 7515264, 12 pages, 2017.

[9] R. Kubacki, S. Lamari, M. Czyzewski, and D. Laskowski, “A broadband left-handed metamaterial microstrip antenna with double-fractal layers," International Journal of Antennas and Propagation, vol. 2017, Article ID 6145865, 6 pages, 2017.

[10] D. Sievenpiper, Lijun Zhang, R. F. J. Broas, N. G. Alexopolous, and E. Yablonovitch, "High-impedance electromagnetic surfaces with a forbidden frequency band," IEEE Transactions on Microwave Theory and Techniques, vol. 47, no. 11, pp. 2059-2074, 1999.

[11] F. Rahmadani and A. Munir, "Microstrip patch antenna miniaturization using artificial magnetic conductor," in 2011 6th International Conference on Telecommunication Systems, Services, and Applications (TSSA), pp. 219-223, Bali, Indonesia, October 2011.

[12] A. Foroozesh and L. Shafai, "Investigation into the application of artificial magnetic conductors to bandwidth broadening, gain enhancement and beam shaping of low profile and conventional monopole antennas," IEEE Transactions on Antennas and Propagation, vol. 59, no. 1, pp. 4-20, 2011.

[13] B. S. Cook and A. Shamim, "Utilizing wideband AMC structures for high-gain inkjet-printed antennas on lossy paper substrate," IEEE Antennas and Wireless Propagation Letters, vol. 12, pp. 76-79, 2013.

[14] H. Mosallaei and K. Sarabandi, "Antenna miniaturization and bandwidth enhancement using a reactive impedance substrate," IEEE Transactions on Antennas and Propagation, vol. 52, no. 9, pp. 2403-2414, 2004.

[15] N. M. Mohamed-Hicho, E. Antonino-Daviu, M. CabedoFabres, and M. Ferrando-Bataller, "A novel low-profile highgain UHF antenna using high-impedance surfaces," IEEE Antennas and Wireless Propagation Letters, vol. 14, pp. 1014-1017, 2015.

[16] A. T. Almutawa and G. Mumcu, "Small artificial magnetic conductor backed log-periodic microstrip patch antenna," IET Microwaves, Antennas \& Propagation, vol. 7, no. 14, pp. 1137-1144, 2013.

[17] R. Carrel, "The design of log-periodic dipole antennas," in 1958 IRE International Convention Record, pp. 61-75, New York, NY, USA, March 1966.

[18] Z. Bayraktar, P. L. Werner, and D. H. Werner, "The design of miniature three-element stochastic Yagi-Uda arrays using particle swarm optimization," IEEE Antennas and Wireless Propagation Letters, vol. 5, pp. 22-26, 2006. 


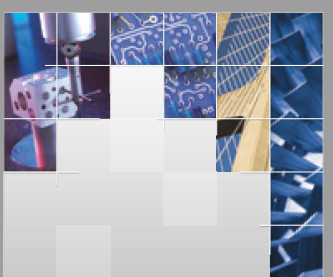

\section{Enfincering}
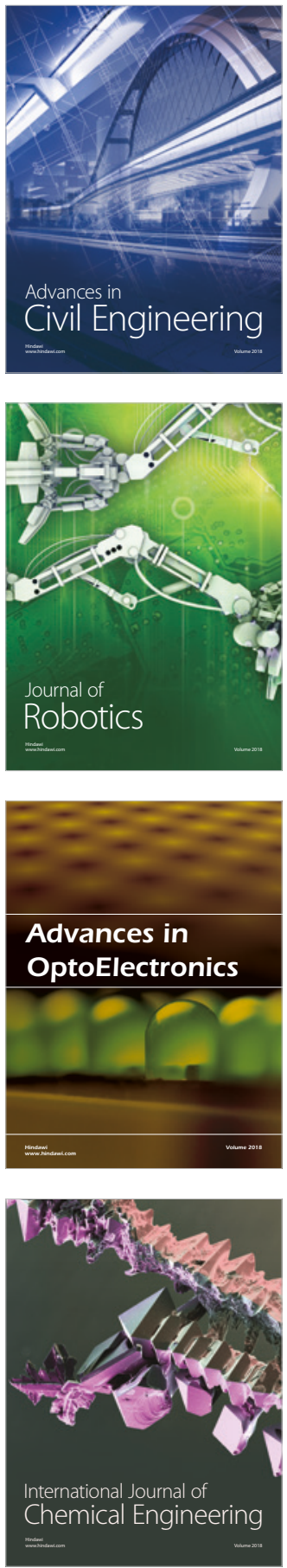

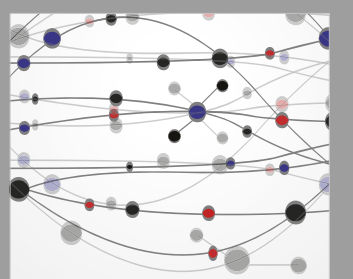

\section{Rotating \\ Machinery}

The Scientific World Journal

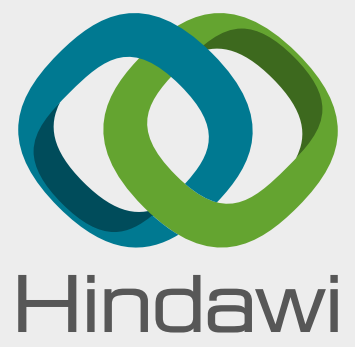

Submit your manuscripts at

www.hindawi.com
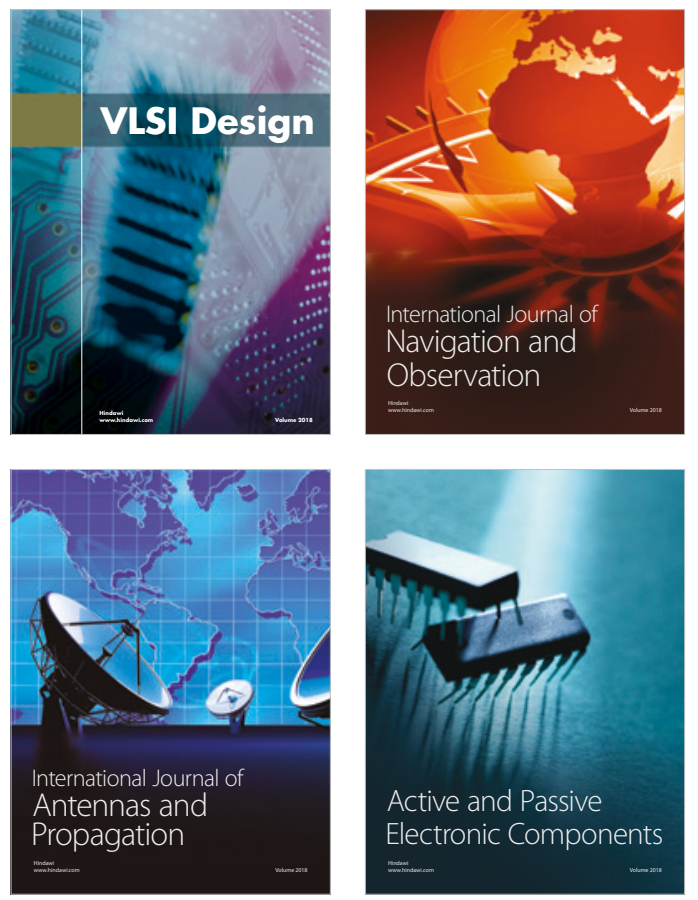
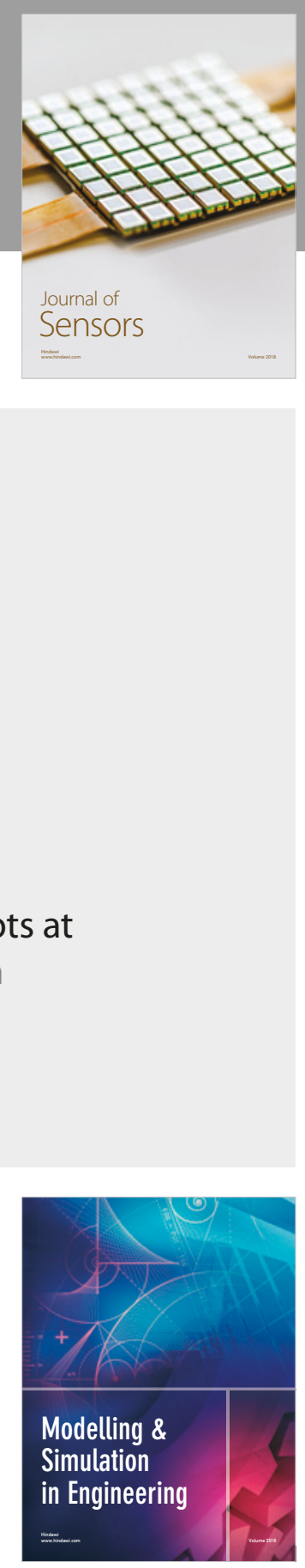

\section{Advances \\ Multimedia}
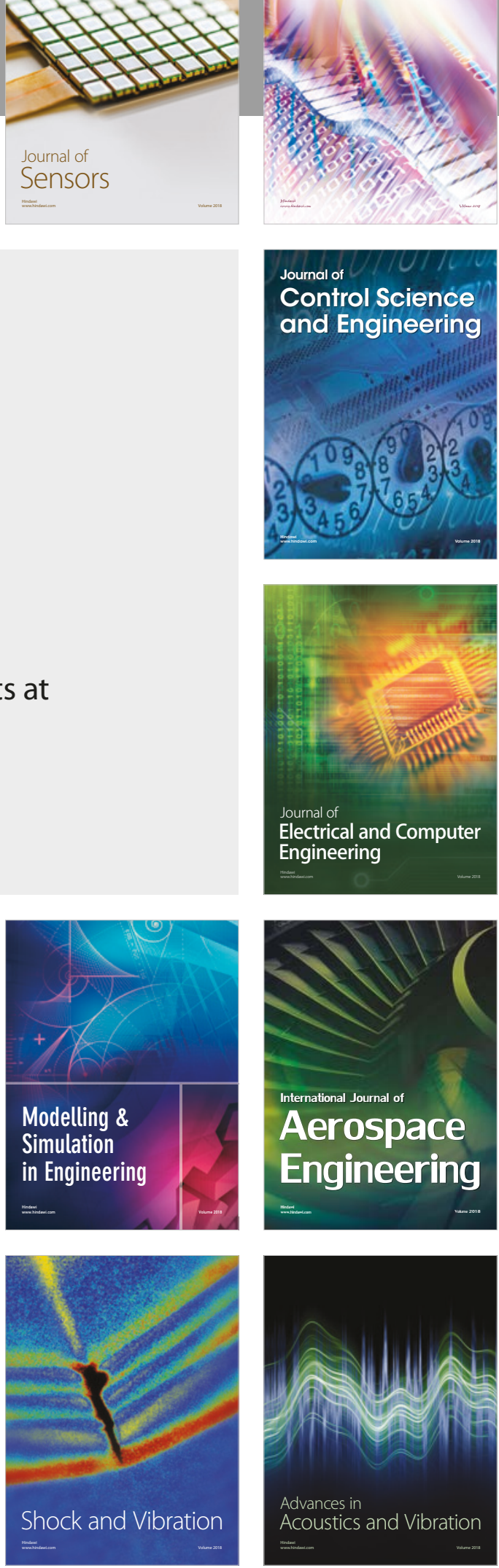